\title{
Nonlinear Models of Miras, Including Time-Dependent Convection
}

\author{
Dale A. Ostlie ${ }^{1}$ and Arthur N. Cox ${ }^{2}$ \\ ${ }^{1}$ Dept. of Physics, Weber State University, Ogden, UT 84408-2508 \\ ${ }^{2}$ Los Alamos National Laboratory, Los Alamos, NM 87545
}

\begin{abstract}
Nonlinear calculations of Mira variable stars of Population I are presented. Each model is $1 \mathrm{M}_{\odot}$, with a luminosity of $5000 \mathrm{~L}_{\odot}$ and an effective temperature near $3000 \mathrm{~K}$. These models incorporate our theory of time-dependent convection, which is based on a convective phase lag formalism and includes spatial averaging of convective eddies from adjacent zonal interfaces. The theory also includes turbulent pressure, energy, and viscosity terms and allows for negative convective luminosities in subadiabatic regions where overshooting occurs.

Results of the present study suggest that based upon the dynamic behavior of the models, fundamental mode pulsations are the preferred mode of oscillation. In particular, we do not obtain the chaotic behavior that has been noted in previous nonlinear studies of the fundamental mode oscillations of Miras.
\end{abstract}

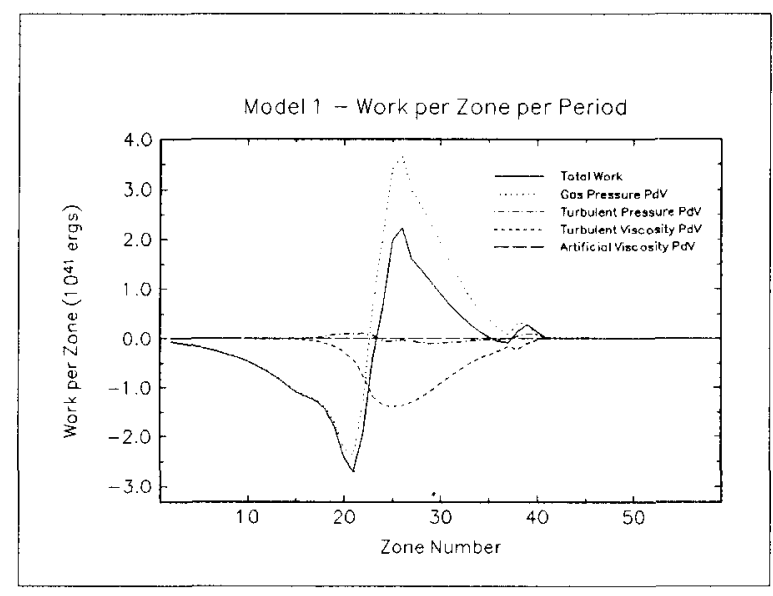

Figure 1 The average amount of work performed per zone per period for a typical fundamental mode model at a limit cycle. 